\title{
Interaction of DODAB with neutral phospholipids and cholesterol studied using fluorescence anisotropy
}

\author{
Graham Hungerford, Adelina L.F. Baptista, Paulo J.G. Coutinho, \\ Elisabete M.S. Castanheira, M. Elisabete C.D. Real Oliveira* \\ Departamento de Física, Universidade do Minho, Campus de Gualtar, 4710-057 Braga, Portugal
}

Received 29 June 2005; received in revised form 3 November 2005; accepted 10 November 2005

Available online 27 December 2005

\begin{abstract}
Liposomes composed of cationic lipids and neutral phospholipids have been used as vehicles for cellular delivery of DNA and drug delivery. The aim of this study is to get a better understanding of phospholipid-cationic lipid interactions that is essential for the development of this potential. We have studied the interaction between cationic lipid liposomes primarily composed of dioctadecyldimethylammonium bromide (DODAB) with two different phospholipids, dioleoyl phosphatidylethanolamine (DOPE) and soybean phosphatidylcholine (PC), as well as with cholesterol. The effect of these components on the liposome's physical properties (microviscosity and polarity) was accessed by studying, both the steady-state and time-resolved, fluorescence anisotropy of the dye Nile Red. This information combined with analysis of the steady-state emission and fluorescence lifetime of Nile Red using the different lipids and cholesterol provided information concerning the polarity and hydration level changes in these vesicle systems along with organisational information.
\end{abstract}

(C) 2005 Elsevier B.V. All rights reserved.

Keywords: DODAB; DOPE; Cholesterol; PC; Nile Red; Fluorescence anisotropy

\section{Introduction}

Dioctadecyldimethylammonium bromide (DODAB) is a long chain cationic surfactant that, in excess of water, self-assembles above the gel to crystalline phase transition temperature $\left(T_{\mathrm{m}}\right)$ into a giant closed bilayer vesicle structure $[1,2]$. The structural organisation of this type of amphiphile depends on the concentration, vesicle preparation method, solvent composition, temperature and any further additives [2,3]. A significant number of studies made with cationic lipids and DNA have reported that the key role of the cationic lipid is to provide an electrostatic attraction between the positively charged liposomes and the negatively charged DNA molecule [4-13]. Cationic liposomes designed for nucleic acid delivery generally are intrinsically unstable and undergo a structural transition upon interacting with the cells $[4,14]$. Membrane

Abbreviations: $\mathrm{Ch}$, cholesterol; DODAB, dioctadecyldimethylammonium bromide; DOPE, dioleoyl phosphatidylethanolamine; NR, Nile Red; PC, soybean phosphatidylcholine

* Corresponding author. Tel.: +351 253 604325; fax: +351 253678981 .

E-mail address: beta@ fisica.uminho.pt (M.E.C.D.R. Oliveira). conformational instability, which appears to be essential to their delivery function, was found to be enhanced by inclusion of a helper lipid into the cationic liposomes [7-9,12,13,15-19]. In contrast, liposomes used for drug delivery are required to be intrinsically stable in order to retain their contents $[20,21]$.

As phospholipid-cationic lipid interactions are known to influence the vesicle properties $[14,17,22]$, studies were performed to ascertain the effect of the cholesterol and the phospholipids, DOPE and PC, on DODAB cationic vesicles in aqueous solution. This involved both steady-state and time-resolved fluorescence anisotropy in order to evaluate the effect of these additives on the liposome's physical properties. While some work has made use of steady-state fluorescence spectroscopy to characterise the properties of the cationic liposomes (hydration, polarity and fluidity) $[11,18,23]$, to our knowledge time-resolved anisotropy has been scantly employed.

The choice of probe for this work, Nile Red, is hydrophobic in nature with a low solubility and fluorescence in water and has demonstrated its value in the study of biological membranes [24,25]. It is highly solvatochromic and both its steady-state and time-resolved fluorescence emission properties 
are strongly medium dependent [26-28]. It usually exhibits an increase in fluorescence yield with decreasing solvent polarity accompanied by a blue shift in the peak emission. By studying the fluorescence anisotropy of Nile Red, it has proved possible to elucidate information concerning the polarity and hydration level changes in vesicle systems [27].

\section{Experimental}

\subsection{Materials}

Soybean phosphatidylcholine (PC), cholesterol (Ch) and dioleoyl phosphatidylethanolamine (DOPE) were purchased from Sigma. Dioctadecyldimethylammonium bromide (DODAB) was purchased from Tokyo Kasei and the solvatochromic probe Nile Red [9-(diethylamino)-5Hbenzo $[\alpha]$ phenoxazin-5-one] (NR) from Aldrich. All were used as received and their structures are depicted below (hydrogens omitted).

\subsection{Fluorescence measurements}

The steady-state fluorescence measurements were performed using Spex Fluorolog 2 spectrofluorimeter. Polarised emission spectra were recorded using Glan-Thompson polarisers. All spectra were corrected for the instrumental response of the system. The time-resolved fluorescence measurements were performed, both at room temperature and $55^{\circ} \mathrm{C}$, using a singlephoton counting spectrometer equipped with pulsed nanosecond LED excitation heads (HORIBA, Jobin Yvon, IBH Ltd., Glasgow, Scotland) with the equipment running in reverse mode because of the high repetition rate $(800 \mathrm{kHz})$. The detection of the fluorescence, wavelength selected using an interference filter or when low intensities were encountered a cutoff filter, was made using a Hamamatsu R2949 photomultiplier. The fluorescence lifetime data were measured to 10,000 counts in the peak, unless otherwise indicated. The instrumental response function was recorded sequentially using a scattering solution and a time calibration of $23 \mathrm{ps} / \mathrm{channel}$, giving a nominal time resolution of

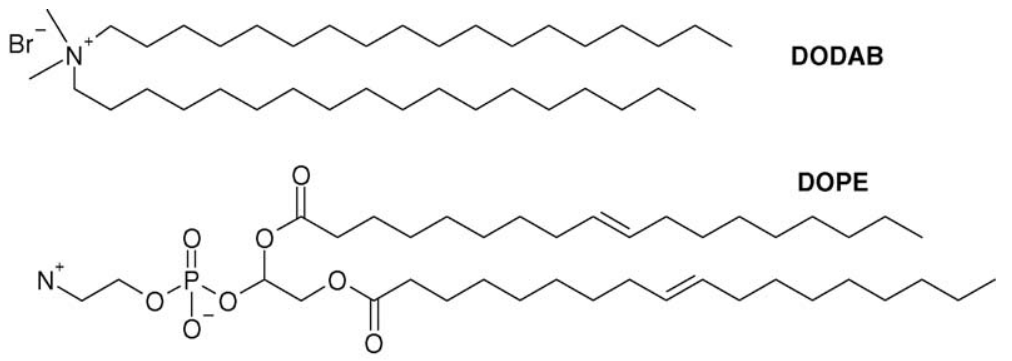

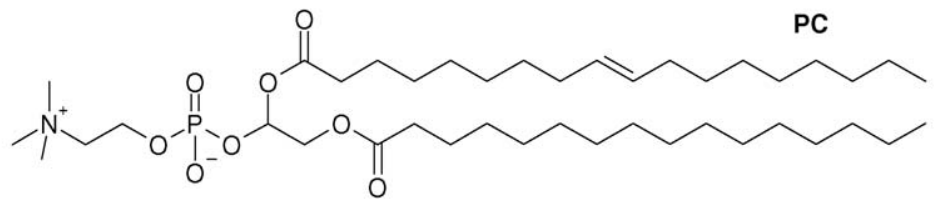<smiles>CC(C)CCCC(C)C1CCC2C3CC=C4CC(O)CCC4(C)C3CCC12C</smiles><smiles>CCN(CC)C1=CC2OC3=CC(=O)C4C=CC=CC4=C3N=C2C=C1</smiles>

\subsection{Vesicle preparation}

Lipid films were prepared from DODAB stock solutions in chloroform, with DOPE, $\mathrm{PC}$ or $\mathrm{Ch}$ at several molar ratios, by evaporation of solvent under a nitrogen stream. Vesicles were formed by hydration of lipids with an aqueous buffer solution (20 mM HEPES and $10 \mathrm{mM} \mathrm{NaCl}$ ), at $60^{\circ} \mathrm{C}$ (above the DODAB transition temperature), followed by sonication in a bath (Heat Systems W-225R), to obtain optically clear solutions. The final DODAB concentration was $1 \mathrm{mM}$. Nile Red $\left(10^{-6} \mathrm{M}\right)$ was introduced to the final vesicle solution by injection of $10 \mu \mathrm{L}$ of a $3 \times 10^{-4} \mathrm{M}$ stock solution of the dye in ethanol. The vesicle solutions were cooled to room temperature and stored for $24 \mathrm{~h}$ prior to measurement.
100 ps after reconvolution. Data were analysed by using a sum of exponentials, employing a non-linear least squares reconvolution analysis from HORIBA, Jobin Yvon, IBH Ltd., of the form:

$I(t)=\sum_{i=1}^{n} \alpha_{i} \exp \left(\frac{-t}{\tau_{i}}\right)$

The pre-exponential factors $\left(\alpha_{i}\right)$ are shown normalised to 1 and the errors are taken as three standard deviations. The goodness of fit was judged in terms of both a Chi-squared $\left(\chi^{2}\right)$ value and weighted residuals. Time-resolved anisotropy decays were analysed making use of the impulse response function (IBH DAS6 software). 


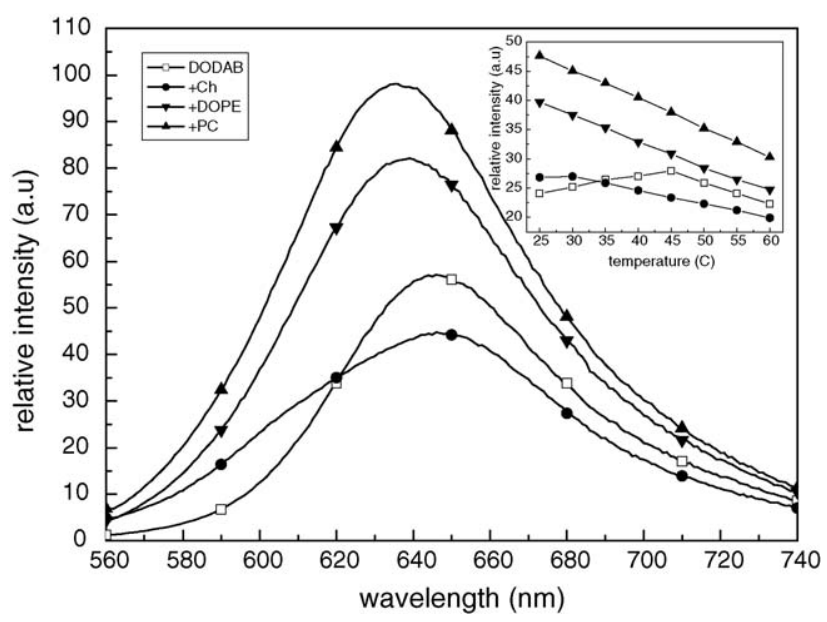

Fig. 1. Emission spectra of Nile Red in the DODAB + (Ch, DOPE or PC) systems for a molar ratio $1: 1\left(\lambda_{\text {exc }}=550 \mathrm{~nm}\right.$, the peak absorption $)$. The inset shows the total fluorescence intensity with temperature.

\section{Results and discussion}

\subsection{Fluorescence emission}

The steady-state fluorescence spectra for Nile Red (NR) incorporated in the DODAB/lipid systems in molar ratio 1:1 is given in Fig. 1. The effect of phospholipids (PC, DOPE) is a reduction of polarity and probably a lower level of hydration in the vesicle interface, where NR is located. Similar trends are seen in the excitation spectra (not shown). The effect of Ch is different and an enhancement on the blue side of the spectrum is observed by the manifestation of a shoulder, but this is accompanied by an overall decrease in the fluorescence quantum yield. The excitation spectrum also exhibits a corresponding shoulder.
The effect of increasing temperature is given as an inset in Fig. 1. This shows the variation of the total fluorescence intensity $\left(I_{\text {total }}\right)$ with temperature. For DODAB alone a maximum is observed at the phase transition temperature $\left(\mathrm{ca} .45^{\circ} \mathrm{C}\right.$ ), after which a steady decrease in intensity is seen. From this data it appears that NR relocates as the temperature increases in the gel state to a less polar environment-more to the interior, giving rise to an increase in fluorescence intensity, due to less water penetration. In the liquid-crystalline phase the NR environment appears more constant and the observed decrease in the fluorescence intensity with temperature follows the normal trend of an increase in non-radiative processes with temperature. The latter trend is also observed in the mixed vesicle systems, which are observed to be in the liquid-crystalline phase.

To compliment the steady-state observations time-resolved fluorescence measurements were performed. These were carried out using two excitation wavelengths (to check for NR partitioning by preferentially exciting NR in non-polar $(490 \mathrm{~nm})$ or more polar $(590 \mathrm{~nm})$ environments), both below and above the DODAB phase transition $\left(T_{\mathrm{m}}\right)$ temperature. The rationale was that is the data recovered appeared excitation wavelength independent then the NR environment could be considered homogeneous, while if the lifetime data exhibited an excitation wavelength dependency then partitioning of the NR was likely to have occurred.

Generally the decays were found to be multiexponential and for the sake of simplicity the recovered lifetimes are also represented by an average value $\left(\tau_{\text {ave }}\right)$, given in Table 1 . The fact that negative pre-exponential factors were recovered is indicative of the occurrence of an excited state process, which we shall discuss later. The $\tau_{\text {ave }}$ value is useful in providing an initial indication concerning the NR environment. For all the systems, with the noticeable exception of DODAB $+\mathrm{Ch}$, and perhaps $\mathrm{DODAB}+\mathrm{DOPE}$ at room temperature, the average

Table 1

Time-resolved data from Nile Red in the different systems at room temperature (RT, ca. $25^{\circ} \mathrm{C}$ ) and $55^{\circ} \mathrm{C}$ and two excitation wavelengths

\begin{tabular}{|c|c|c|c|c|c|c|c|c|c|c|c|}
\hline System & Temp. & $\lambda_{\text {exc }}(\mathrm{nm})$ & $\lambda_{\mathrm{em}}(\mathrm{nm})$ & $\tau_{1}(\mathrm{~ns})$ & $\tau_{2}(\mathrm{~ns})$ & $\tau_{3}(\mathrm{~ns})$ & $\alpha_{1}$ & $\alpha_{2}$ & $\alpha_{3}$ & $\tau_{\text {ave }}(\mathrm{ns})$ & $\chi^{2}$ \\
\hline \multirow[t]{4}{*}{ DODAB } & RT & 490 & 650 & $3.54 \pm 0.08$ & $2.75 \pm 0.45$ & & 0.49 & 0.51 & & 3.1 & 1.11 \\
\hline & & 590 & 650 & $3.51 \pm 0.06$ & $2.53 \pm 0.09$ & & 0.41 & 0.59 & & 2.9 & 1.08 \\
\hline & $55^{\circ} \mathrm{C}$ & 490 & $>570$ & $3.58 \pm 0.95$ & $2.05 \pm 0.03$ & $0.78 \pm 0.61$ & 0.03 & 0.82 & -0.15 & 1.9 & 1.11 \\
\hline & & 590 & 650 & $4.76 \pm 2.65$ & $2.10 \pm 0.01$ & $0.23 \pm 0.06$ & 0.01 & 0.65 & -0.34 & 1.5 & 1.15 \\
\hline \multirow[t]{4}{*}{ DODAB + DOPE } & RT & 490 & 650 & $5.60 \pm 2.20$ & $3.56 \pm 0.06$ & $2.35 \pm 0.64$ & 0.03 & 0.62 & -0.35 & 3.2 & 1.17 \\
\hline & & 590 & 650 & $3.79 \pm 0.06$ & $1.89 \pm 0.24$ & $1.60 \pm 0.32$ & 0.34 & 0.33 & -0.33 & 2.4 & 1.09 \\
\hline & $55^{\circ} \mathrm{C}$ & 490 & 650 & $2.21 \pm 0.01$ & $0.78 \pm 0.07$ & & 0.68 & -0.32 & & 1.8 & 1.17 \\
\hline & & 590 & 650 & $2.66 \pm 0.81$ & $1.84 \pm 0.06$ & $0.85 \pm 0.60$ & 0.12 & 0.69 & -0.19 & 1.8 & 1.07 \\
\hline \multirow[t]{4}{*}{$\mathrm{DODAB}+\mathrm{PC}$} & RT & 490 & 650 & $3.96 \pm 0.05$ & $2.38 \pm 0.21$ & $1.10 \pm 0.31$ & 0.67 & 0.24 & -0.09 & 3.3 & 1.10 \\
\hline & & 590 & 650 & $4.22 \pm 0.63$ & $3.61 \pm 2.91$ & $1.06 \pm 0.15$ & 0.17 & 0.65 & -0.18 & 3.3 & 1.11 \\
\hline & $55^{\circ} \mathrm{C}$ & 490 & 650 & $3.60 \pm 0.51$ & $2.49 \pm 0.39$ & $1.00 \pm 0.05$ & 0.05 & 0.61 & -0.34 & 2.0 & 1.26 \\
\hline & & 590 & 650 & $3.86 \pm 1.50$ & $2.38 \pm 0.03$ & $0.72 \pm 0.42$ & 0.04 & 0.77 & -0.19 & 2.1 & 1.19 \\
\hline \multirow[t]{4}{*}{$\mathrm{DODAB}+\mathrm{Ch}$} & RT & 490 & 650 & $8.87 \pm 2.70$ & $5.68 \pm 0.96$ & $2.11 \pm 0.81$ & 0.07 & 0.80 & -0.13 & 5.4 & 1.05 \\
\hline & & 590 & 650 & $5.89 \pm 0.06$ & $3.05 \pm 0.24$ & & 0.50 & 0.50 & & 4.5 & 1.09 \\
\hline & $55^{\circ} \mathrm{C}$ & 490 & $>570$ & $5.28 \pm 0.09$ & $3.30 \pm 0.12$ & $0.54 \pm 0.39$ & 0.21 & 0.67 & -0.12 & 3.4 & 1.23 \\
\hline & & 590 & $>615$ & $4.00 \pm 0.15$ & $2.17 \pm 0.25$ & $0.18 \pm 0.15$ & 0.12 & 0.35 & 0.53 & 1.3 & 1.02 \\
\hline
\end{tabular}

The emission was selected using either interference or cutoff filters. 
lifetime values within each system do not exhibit any significant dependency on excitation wavelength. Thus, we shall, as a first approximation, consider that the environment probed by NR is homogeneous. This assumption will not be applied to $\mathrm{DODAB}+\mathrm{Ch}$, where there is a great likelihood that NR partitions between two (or more) locations. The $\tau_{\text {ave values at }}$ higher temperatures are similar for all systems (DODAB $+\mathrm{Ch}$ excluded) and approximately $60 \%$ those of the lower temperature showing an increase in non-radiative deactivation pathways. The trend below the $T_{\mathrm{m}}$ for DODAB, shows a minor increase from DODAB alone, to DODAB with the addition of DOPE to DODAB with PC. A slight wavelength dependency is seen for DODAB with DOPE at room temperature may hint at some partitioning of NR between different environments.

\subsection{Anisotropy analysis}

If we consider the steady-state anisotropy for NR in the different systems, shown in Fig. 2, it is clear that there is a wavelength dependency. We have previously analysed such behaviour for NR in mixed lipid systems by considering a dual emission from an initially excited state and a solvent relaxed state [27]. Here it seems pertinent to do so again and the fluorescence spectra obtained at vertical and horizontal polarisation (with vertically polarised excitation) were fitted to a sum of two lognormal functions, one for each emitting state, with the same width and skewness parameters. The steady-state fluorescence anisotropies for the two states ( 1 and 2 ) were then calculated from:

$r_{1}=\frac{\left(A_{\mathrm{VV}}\right)_{1}-\left(A_{\mathrm{VH}}\right)_{1}}{\left(A_{\mathrm{VV}}\right)_{1}+2\left(A_{\mathrm{VH}}\right)_{1}} \quad r_{2}=\frac{\left(A_{\mathrm{VV}}\right)_{2}-\left(A_{\mathrm{VH}}\right)_{2}}{\left(A_{\mathrm{VV}}\right)_{2}+2\left(A_{\mathrm{VH}}\right)_{2}}$

The emission intensity fraction of the lower wavelength excited state is:

$f_{1}=\frac{\left(A_{\mathrm{VVV}}\right)_{1}+2\left(A_{\mathrm{VH}}\right)_{1}}{\left(A_{\mathrm{VVV}}\right)_{1}+2\left(A_{\mathrm{VH}}\right)_{1}+\left(A_{\mathrm{VV}}\right)_{2}+2\left(A_{\mathrm{VH}}\right)_{2}}$

where $A_{\mathrm{VV}}$ and $A_{\mathrm{VH}}$ are the maximum emission intensities of the spectra obtained with vertical and horizontal emission

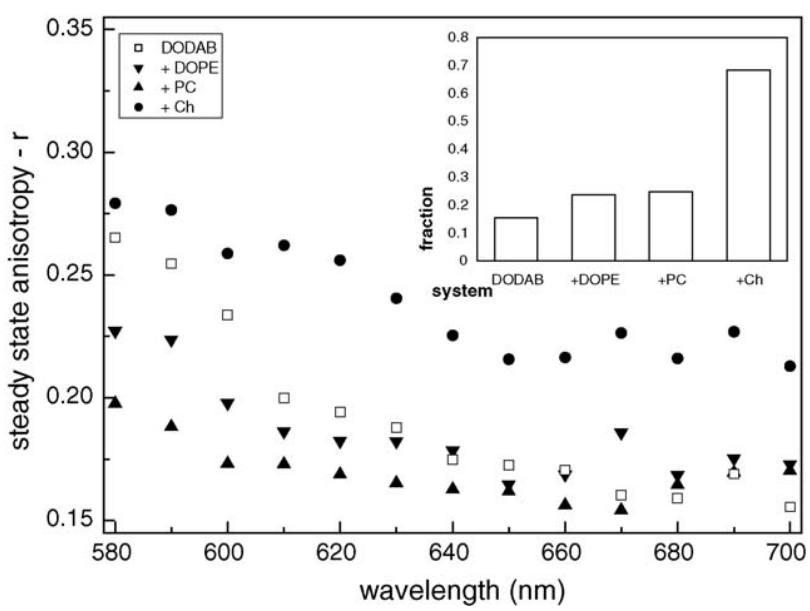

Fig. 2. Variation in NR anisotropy with emission wavelength for NR in the $\mathrm{DODAB}+(\mathrm{Ch}, \mathrm{DOPE}$ or PC) systems for a molar ratio $1: 1$. Inset is the fraction of fluorescence attributed to excited state 1. polarisation, respectively (for excitation with vertical polarised light).

The inset of Fig. 2 shows the fraction of the state 1 for the different samples. On addition of DOPE or PC there is only a slight increase in the proportion of this excited state over DODAB alone and the values are close to those seen using NR in egg PC [29]. The significant difference in the data appears to be that for DODAB $+\mathrm{Ch}$, however it should be kept in mind that the origin of the emission in this case is from differently located NR and not from an initial and relaxed state (although each location may have emissions from both). The use of extra components did not significantly improve matters and in order to avoid an over parameterisation of the data, a sum of two lognormal functions was used throughout to describe the data. Hence for simplicity the states will be named 1 and 2 throughout and their origin dependent on the system studied.

The effect of the addition of different quantities of additive to DODAB is demonstrated in Fig. 3, where this spectral decomposition was employed [27]. The lower wavelength emission shows that the behaviour on addition of DOPE or PC is similar; a decrease in peak emission wavelength accompanied by a reduction in anisotropy. State 2 also shows a similar behaviour, except that the anisotropy does not change. In both cases the addition of $\mathrm{Ch}$ initially follows this trend, but then produces (at $50 \%$ ) a dramatic shift to longer wavelengths. For state 1 the

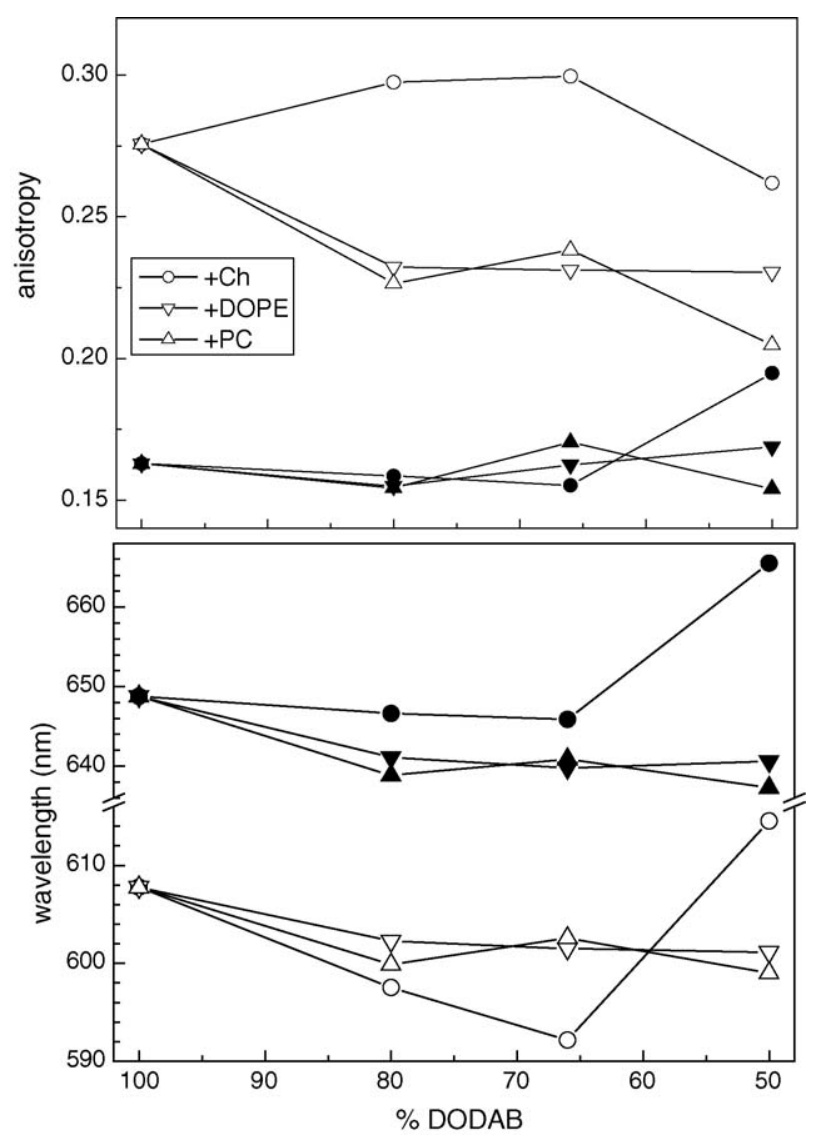

Fig. 3. Position of the peak emission and associated anisotropies for NR in DODAB with different quantities of additives. Open symbols relate to state 1 and closed to state 2 . 
steady-state anisotropy appears higher for all DODAB/Ch fractions, than for the other systems, while no difference in this value is noted for state 2, for DODAB/Ch fractions lower than 0.25 . The higher value of $r_{1}$ for DODAB + Ch system suggest either some resistance to rotational relaxation motion (more viscous environment) or a restricted environment for NR. The above observations can be explained, if initially there is an even distribution of Ch between the lipids, which on Ch enrichment leads to the formation of lipid-cholesterol condensed complexes [30]. Overall this would explain the blue shift in the Nile Red emission spectrum as the lipid packing changes and the dye is less accessible to the aqueous region. At the higher $\mathrm{Ch}$ concentrations the cholesterol can self associate to form crystalline domains [31], which expel the probe to a more polar environment. This effect is more pronounced in the wavelength data, but is also perceptible in the anisotropy data. Although simplistic this form of analysis shows its merits in elucidating information concerning these difficult systems.

In order to obtain further data time-resolved anisotropy measurements were performed at two excitation wavelengths to attempt to excite different environmental distributions of NR $(490 \mathrm{~nm}$ NR in non-polar and $590 \mathrm{~nm}$ for NR in more polar environments) in the mixed systems and the outcome is represented in Fig. 4. This shows that, at room temperature, the use of different excitation wavelengths uncovers different rotational correlation times (Fig. 4a). This is especially pronounced in the case of the sample with added $\mathrm{Ch}$, which in contrast to the other samples exhibits a shorter time with excitation at $590 \mathrm{~nm}$. This has been studied elsewhere as a function of $\mathrm{Ch}$ concentration [32]. In general, at the elevated temperature, all the rotational correlation times are on a similar timescale with a slightly higher value recovered for the longer wavelength excitation, with a slight difference noted for DODAB + Ch. This occurs because the rotational correlation times reflect the viscosity of the microenvironment and at $55^{\circ} \mathrm{C}$, all the systems are in the liquid-crystalline phase and exhibit similar viscosities. To elucidate confinement of the probe we calculated the rotational hindrance via Eq. (4):

$2\left(\frac{r_{\infty}}{r_{0}}\right)^{1 / 2}=\cos ^{2} \theta+\cos \theta$

where $\theta$ is the semi-angle of a cone in which the fluorophore can freely rotate.

Fig. $4 \mathrm{~b}$ shows the ability of the NR to rotate calculated from the initial and residual anisotropies ( $r_{0}$ and $r_{\infty}$, respectively) using the wobbling in cone approximation [33] via Eq. (4). For the sake of clarity the vertical axis represents $90^{\circ}$ minus the semi-angle, i.e. the larger the value shown the greater the hindrance. It can be seen from this figure that, with the exception of the Ch sample, at $55^{\circ} \mathrm{C}$ the NR in a more non-polar environment is completely free to rotate. However, at the same temperature, monitoring the NR emission with an excitation wavelength of $590 \mathrm{~nm}$, shows a degree of hindrance associated with the head group packing. The inclusion of additives to DODAB produces a slightly more restrictive environment for the NR. The corresponding room temperature data show, in the case of DODAB
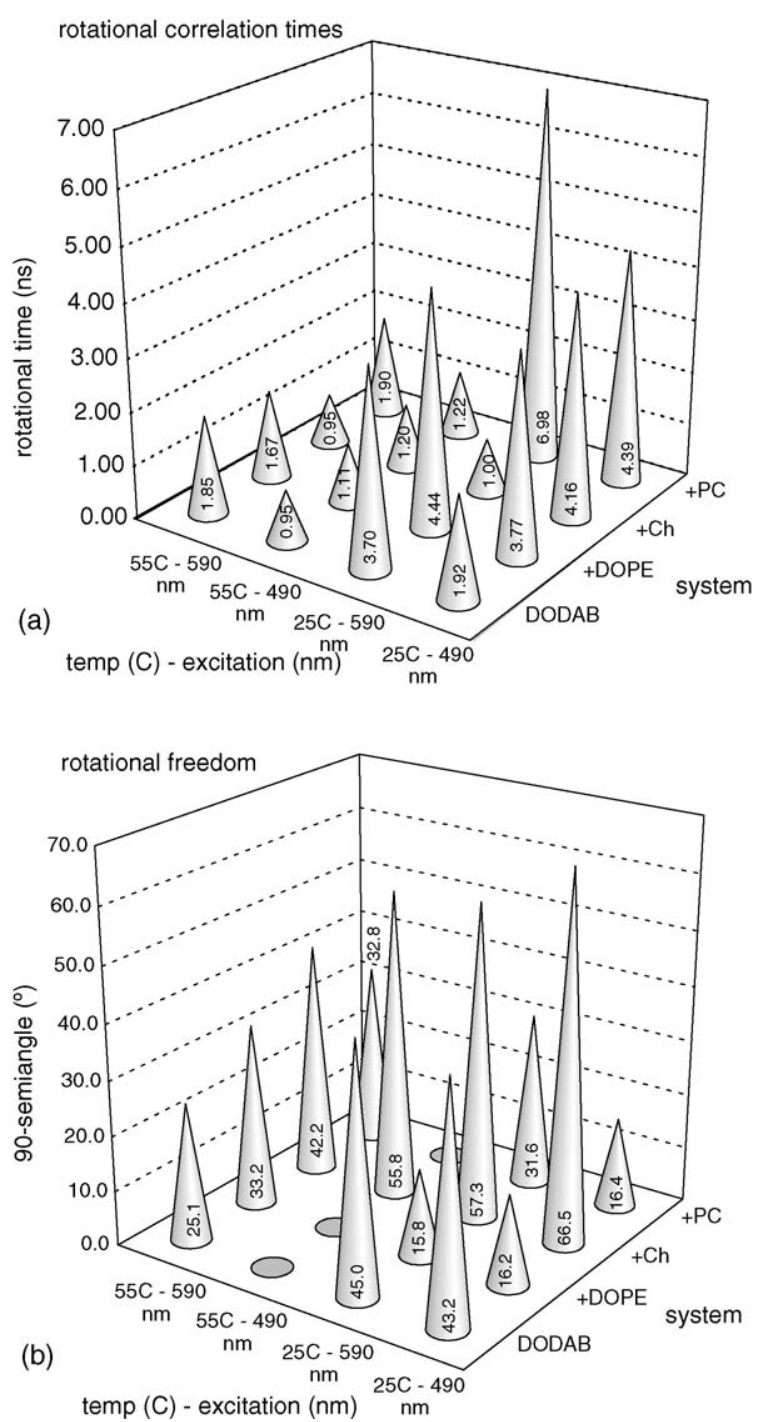

Fig. 4. Time-resolved anisotropy data for NR in the DODAB systems at different temperatures and excitation wavelengths, giving (a) the rotational correlation times and (b) the rotational hindrance calculated from Eq. (4). For clarity in (b) the $y$-axis gives $90^{\circ}$-the semicone angle, i.e. larger values indicate greater hindrance.

alone, that the NR encountered a similarly restrictive environment, which relates to the fact that at this temperature the system is in the gel state. The system with DOPE exhibits a similar, but smaller, hindrance for both excitation wavelengths. It should be noted that the system is in the liquid-crystalline phase, as is DODAB + PC. Here a difference can be observed between the two excitation wavelengths. Using $490 \mathrm{~nm}$ the value is similar to that with DOPE, but at the higher excitation wavelength the environment is more restrictive, although not as restrictive as with DODAB alone. This indicates that $\mathrm{PC}$ packs better than DOPE in this region. Again the results with the addition of $\mathrm{Ch}$ are markedly different.

Cholesterol is known to increase the microviscosity in vesicles [34] and this is supported by the steady-state anisotropy data (see Fig. 3), although only at high Ch concentrations considering state 2 . The time-resolved data indicate, however, that the viscosity probed by NR (as seen from the rotational correla- 


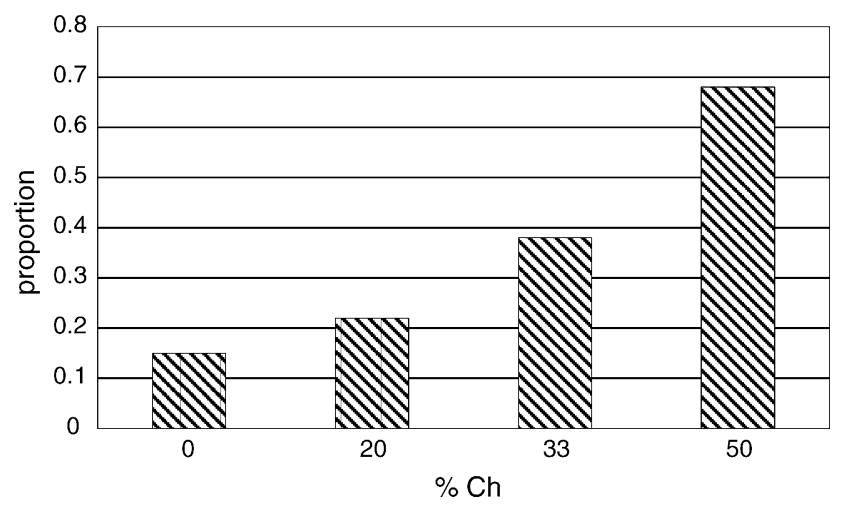

Fig. 5. Proportion of emission intensity associated with state 1 for NR in DODAB with increasing quantity of $\mathrm{Ch}$.

tion time [33]) on the whole is no greater than that found in the DODAB systems with other additives. The major difference is observed in the hindrance of the NR rotation, which is indicative of NR located, either in the head group region or in another confining environment.

In order to elucidate further information concerning the effect of $\mathrm{Ch}$ enrichment, progressive addition of $\mathrm{Ch}$ was made to the DODAB vesicles and the steady-state spectra analysed using Eq. (3). The fraction associated with the lower wavelength emitting state (state 1) is represented in Fig. 5. This shows that at lower concentrations the major emission arises from state 2, whose emission wavelength (Fig. 3) remains more or less constant. The fraction of emission associated with state 1 does not increase much until a concentration of $33 \% \mathrm{Ch}$ is reached, although NR reports on an increasingly non-polar environment (Fig. 3). At the $50 \%$ addition of $\mathrm{Ch}, \mathrm{NR}$ emission reports on a polarity increase for both emissions and now the major fraction is that associated with the shorter wavelength (state 1) emission. This data also supports the fact, that the increase in Ch concentration effects the organisation of the bilayer by first forming condensed complexes with the cationic lipids and then, at higher $\mathrm{Ch}$ concentrations, crystalline domains $[30,35]$.

\subsection{Excited state behaviour}

Previous analysis of NR in membrane systems have analysed the emission as originating from two states [27,29]. A model employed by Krishna [29], which consists of a simple two-state model is shown in Scheme 1, where $\mathrm{A}^{*}$ and $\mathrm{B}^{*}$ represent the initially excited and newly formed excited state species, with the possibility of a reversible reaction.

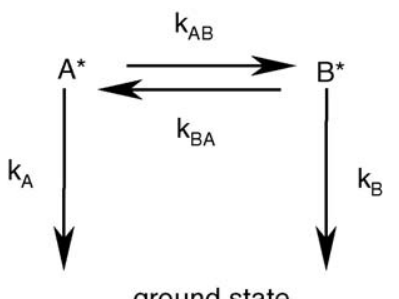

ground state

Scheme 1
As the fuller decay time analysis (Table 1) recovered negative pre-exponential components, thus indicating the presence of an excited state process, along with the fact that the decomposition technique [27] applied to the anisotropy data provided acceptable fits, is supportive of using this type of model. However, as at times the sum of three decay components is required to provide a satisfactory fit to the data, shows that the process is probably more complex and raises the possibility that NR is also distributed between different local environments. This is especially evident in the system with $\mathrm{Ch}$, where it is clear that the simple two state solvent relaxed model is not sufficient. NR is well known for its environmental sensitivity [36] and ability to report on different environments [26]. The results obtained here are indicative of the presence of more than one microenvironment and decomposing the NR emission spectrum into a sum of two individual components a difference of $\sim 40 \mathrm{~nm}$ is obtained. This is greater than that (in the order of $10 \mathrm{~nm}$ ) previously obtained for initially excited and solvent relaxed states in vesicles [29], although shifts, of a similar magnitude to those observed here, have been reported for solvent relaxation of this dye [27] and also for Coumarin 153 [37]. The latter has also exhibited solvent relaxation in mixed lecithin-taurocholate mixed micelles [38].

Although not possible to resolve in terms of wavelength, because of the use of filters, calculations were attempted to obtain some of the rate constants by assuming the possibility of a two-state model or even the presence of two, two-state models, to mimic two environments. In the case of an irreversible process, values of $k_{\mathrm{AB}}$ were obtained using both combinations of $\tau_{1}$ and $\tau_{2}$, and $\tau_{1}$ and $\tau_{3}$. In both cases $\tau_{1}$ was associated with $\mathrm{B}^{*}$. Similar calculations were done considering a reversible process to obtain $\left(k_{\mathrm{B}}+k_{\mathrm{BA}}\right)^{\mathrm{app}}$ [29]. However, from our decomposition the intensity of the lower wavelength spectrum (inset Fig. 2) appears to be the minor one, which can indicate that the excited state is irreversible [29]. A complete analysis of the lifetime values is difficult and would require more thorough data, however rate constants in the range $0.1-0.3 \mathrm{~ns}^{-1}$ can be obtained using $\tau_{1}$ and $\tau_{2}$ (an exception is 0.8 obtained for DODAB + DOPE, $490 \mathrm{~nm}$ excitation, $55^{\circ} \mathrm{C}$ ), while higher values 0.3 to 1.7 , exception 4.1 for DODAB at $590 \mathrm{~nm}$ excitation, $55^{\circ} \mathrm{C}$ ) are recovered using $\tau_{1}$ and $\tau_{3}$.

The time-resolved data show the complexity of these systems, however it is important to note that the simple anisotropy and time-resolved analysis have proved useful analytical tools, although it is acknowledged that the results obtained may represent an average of a combination of processes.

\section{Conclusion}

The utilisation of steady-state and time-resolved anisotropy coupled with the solvatochromic probe Nile Red has proved useful in characterising the different DODAB mixed systems. On addition of DOPE, or DOPC to DODAB there is an homogeneous mixing of the lipids and a two-state model involving solvent relaxation is adequate to describe the system. A different behaviour is elucidated by the inclusion of cholesterol, where a progression from even distribution to complex and domain formation with cholesterol enrichment of the DODAB 
vesicles can be observed. Here a two-state model may also be employed, but describing the partitioning of NR between different environments.

\section{References}

[1] A.M. Carmona-Ribeiro, Chem. Soc. Rev. 21 (1992) 209.

[2] E. Feitosa, W. Brown, Langmuir 13 (1997) 4810.

[3] E. Feitosa, P.C.A. Barreleiro, G. Olofsson, Chem. Phys. Lipids 105 (2000) 201.

[4] P. Pires, S. Simões, S. Nir, R. Gaspar, N. Duzgunes, M.C. Pedroso de Lima, Biochim. Biophys. Acta 1418 (1999) 71.

[5] H. Farhood, N. Serbina, L. Huang, Biochim. Biophys. Acta 1235 (1995) 289.

[6] P.C.A. Barreleiro, G. Olofsson, P. Alexandrisdis, J. Phys. Chem. B 104 (2002) 7795 .

[7] R.W. Malone, P.L. Felgner, I.M. Verma, Proc. Natl. Acad. Sci. U.S.A. 86 (1989) 6077.

[8] P.L. Felgner, T.R. Gadek, M. Holm, R. Roman, H.W. Chan, M. Wenz, J.P. Northrop, G.M. Ringold, M. Danielsen, Proc. Natl. Acad. Sci. U.S.A. 84 (1987) 7413.

[9] J.H. Felgner, R. Kumar, C.N. Sridhar, C.J. Wheeler, Y.J. Tsai, R. Border, P. Ramsey, M. Martim, P.L. Felgner, J. Biol. Chem. 269 (1994) 2550.

[10] P.C.A. Barreleiro. Equilibrium and kinetics studies of the binding of DNA to cationic lipids, Ph.D. Thesis, University of Lund, Sweden, 2001.

[11] N.J. Zuidam, Y. Barenholz, Biochim. Biophys. Acta 1329 (1997) 211.

[12] S.J. Eastman, C. Siegel, J. Tousignant, A.E. Smoth, S.H. Cheng, R.K. Scheule, Biochim. Biophys. Acta 1325 (1997) 41.

[13] Y.S. Mel'nikova, S.M. Mel'nikov, J.-E. Löfroth, Biophys. Chem. 81 (1999) 125.

[14] K. Romøren, B.J. Thu, N.C. Bols, Ø. Evensen, Biochim. Biophys. Acta 1663 (2004) 127.

[15] H. Ellens, J. Bentz, F.C. Szoka, Biochemistry 25 (1986) 285.

[16] I. Aksentijevich, I. Pastan, Y. Lunardi-Iskandar, R.C. Gallo, M.M. Gottesman, Hum Gene Ther. 7 (1996) 1111.

[17] R.B. Campbell, V.S. Balasubramanian, R.M. Straubinger, Biochim. Biophys. Acta 1512 (2001) 27.
[18] D. Hirsch-Lerner, Y. Barenholz, Biochim. Biophys. Acta 1461 (1999) 47.

[19] L. Ciani, S. Ristori, A. Salvati, L. Calamai, G. Martini, Biochim. Biophys. Acta 1664 (2004) 70.

[20] A.R. Thierry. Liposomal delivery system for biologically active agents, U.S. Patent 6,110,490 (1996).

[21] J. Senior, G. Gregoriadis, Life Sci. 30 (1982) 2123

[22] Y. Zhang, T.J. Anchordoquy, Biochim. Biophys. Acta 1663 (2004) 143.

[23] D. Hirsch-Lerner, Y. Barenholz, Biochim. Biophys. Acta 1329 (1997) 211.

[24] P. Greenspan, S.D. Fowler, J. Lipid Res. 26 (1985) 781.

[25] Ira, G. Krishnamoorthy, J. Phys. Chem. B 105 (2001) 1484.

[26] G. Hungerford, E.M.S. Castanheira, M.E.C.D. Real Oliveira, M.da.G. Miguel, H.D. Burrows, J. Phys. Chem. B 106 (2002) 4061.

[27] P.J.G. Coutinho, E.M.S. Castanheira, M. Céu Rei, M.E.C.D. Real Oliveira, J. Phys. Chem. B 106 (2002) 12841.

[28] N. Ghoneim, Spectrochim. Acta A 56 (2000) 1003.

[29] M.M.G. Krishna, J. Phys. Chem. A 103 (1999) 3589.

[30] H.M. McConnell, A. Radhakrishnan, Biochim. Biophys. Acta 1610 (2003) 159.

[31] R.P. Mason, T.N. Tulenko, R.F. Jacob, Biochim. Biophys. Acta 1610 (2003) 198.

[32] G. Hungerford, A.L.F. Baptista, P.J.G. Coutinho, E.M.S. Castanheira, M.E.C.D. Real Oliveira, J. Fluoresc., in press, doi:10,1007/s10895-0050014-3.

[33] R. Steiner, in: J.R. Lakowicz (Ed.), Topics in Fluorescence Spectroscopy, vol. 2, Plenum Press, New York, 1991, pp. 1-52.

[34] J.R. Lakowicz, Principles of Fluorescence Spectroscopy, Plenum Press, New York, 1983.

[35] S. Koronkiewicz, S. Kalinowski, Biochim. Biophys. Acta 1661 (2004) 196.

[36] T.M.R. Viseu, G. Hungerford, A.F. Coelho, M.I.C. Ferreira, J. Phys. Chem. B 107 (2003) 13300.

[37] S. Arzhantsev, N. Ito, M. Heitz, M. Maroncelli, Chem. Phys. Lett. 381 (2003) 278.

[38] D. Chakrabarty, A. Chakraborty, D. Seth, P. Hazra, N. Sarkar, Chem. Phys. Lett. 412 (2005) 255. 